ARTICLE

\title{
Post-traumatic stress symptoms in COVID-19 survivors: a self- report and brain imaging follow-up study
}

\author{
Yiheng Tu (iD ${ }^{1,2,6}$, Yuqi Zhang ${ }^{2,3,6}$, Yu Li ${ }^{4,6}$, Qing Zhao ${ }^{1,2}$, Yanzhi $\mathrm{Bi}^{1,2}$, Xuejing $\mathrm{Lu}^{1,2}$, Yazhuo Kong ${ }^{2,3}$, Li Wang $\mathbb{D}^{1,2}$, Zhijie Lu (iD ${ }^{5^{凶}}$ and \\ Li Hu (iD ${ }^{1,2}$
}

(c) The Author(s), under exclusive licence to Springer Nature Limited 2021

Previous coronavirus pandemics were associated elevated post-traumatic stress symptoms (PTSS), but the self-report and neurological basis of PTSS in patients who survived coronavirus disease 2019 (COVID-19) are largely unknown. We conducted a two-session study to record PTSS in the COVID-19 survivors discharged from hospitals for a short (i.e., about 3 months, Session 1) to a medium period (i.e., about 6 months, Session 2), as well as brain imaging data in Session 2. The control groups were non-COVID19 locals. Session 1 was completed for 126 COVID-19 survivors and 126 controls. Session 2 was completed for 47 COVID-19 survivors and 43 controls. The total score of post-traumatic stress disorder (PTSD) checklist for DSM-5 (PCL-5) score was significantly higher in COVID-19 survivors compared with controls in both sessions. The PCL-5 score in COVID-19 survivors was positively correlated with the duration after discharge ( $r=0.27, p=0.003$ for Session 1), and increased by 20\% from Session 1 to Session 2 for the survivors who participated both sessions. The increase was positively correlated with individual's test-retest duration $(r=0.46, p=0.03)$. Brain structural volume and functional activity in bilateral hippocampus and amygdala were significantly larger in COVID-19 survivors compared with controls. However, the volumes of the left hippocampus and amygdala were negatively correlated with the PCL-5 score for the COVID-19 survivors. Our study suggests that COVID-19 survivors might face possible PTSS deteriorations, and highlights the importance of monitoring mental wellness of COVID-19 survivors.

Molecular Psychiatry (2021) 26:7475-7480; https://doi.org/10.1038/s41380-021-01223-w

\section{INTRODUCTION}

Coronavirus Disease 2019 (COVID-19) was first identified in Wuhan, Hubei Province, China [1], and the spread of severe acute respiratory syndrome coronavirus 2 became a global pandemic, with more than 150 million cases confirmed, and 3.2 million lives lost in more than 200 countries by the end of April 2021. Although considered as a respiratory disease, COVID-19 has become increasing recognized as neurotropic since patients might experience mild to severe neurological symptoms [2,3]. Using magnetic resonance imaging (MRI), previous studies reported that half of the COVID-19 patients have brain structural abnormalities, including white matter hyperintensities $[4,5]$ and frontal-temporal gray matter volume abnormalities [6].

While the control of epidemic and care of patients are the most crucial tasks, new research is now suggesting that there may be longterm consequences, such as fatigue or muscle weakness, sleep difficulties, anxiety or depression, in those 60 million people who recovered from COVID-19 infections [7]. Crucially, COVID-19 survivors who experienced fear for survival, are vulnerable to develop posttraumatic stress symptoms (PTSS) [8-10]. A recent study found that COVID-19 survivors in Italy suffered severe PTSS shortly after they were discharged (e.g., within 1 month) from the hospital [9]. However, it is still unknown whether COVID-19 survivors suffer longer-term PTSS (e.g., about 3 months to 6 months), and whether their PTSS are associated with evident brain abnormalities.

To tap into the above issues, we conducted a two-session follow-up study using both self-report assessments and brain imaging techniques. In Session 1, we recruited 126 COVID-19 survivors (about 3 months after discharged from hospitals in Wuhan, China) and 126 matched non-COVID-19 controls, and compared their self-report PTSS. In Session 2, 50 COVID-19 survivors (about 6 months after discharged from hospitals) and 43 matched non-COVID-19 controls were recruited from Wuhan, China. Their self-report PTSS, and structural and functional brain MRI data were collected. We hypothesized that COVID-19 survivors (1) would have severe PTSS than the controls; (2) would develop more severe PTSS along with the duration after discharge; (3) would have brain structural and functional abnormalities at key brain regions involved in the processing of PTSS [11] (e.g., hippocampus [12] and amygdala [13]).

\section{MATERIALS AND METHODS} Participants and study design

COVID-19 survivors (i.e., cases with a clinical diagnosis and were discharged from hospitals in Wuhan, China, at the time of the testing)

\footnotetext{
${ }^{1}$ CAS Key Laboratory of Mental Health, Institute of Psychology, Beijing, China. ${ }^{2}$ Department of Psychology, University of Chinese Academy of Sciences, Beijing, China. ${ }^{3}$ CAS Key Laboratory of Behavioral Science, Institute of Psychology, Beijing, China. ${ }^{4}$ Organ Transplantation Department, Third Affiliated Hospital of Second Military Medical University, Shanghai, China. ${ }^{5}$ Department of Anesthesiology, Third Affiliated Hospital of Second Military Medical University, Shanghai, China. ${ }^{6}$ These authors contributed equally: Yiheng Tu, Yuqi Zhang, Yu Li. ${ }^{凶}$ email: Izjwxyz@163.com; huli@psych.ac.cn
} 
and age- and sex-matched non-COVID-19 controls were recruited from the community. The criteria of diagnosis and discharge of patients were based on the Polymerase Chain Reaction result according to World Health Organization guidelines [14]. The ethical protocols for conducting the current study were approved by the ethics committee of the local hospital. All participants provided their informed consent at the beginning of the study.

The present study consisted of two sessions, and the sample sizes were chosen based on recent studies investigating COVID-19, as well as the ongoing situation of the pandemic. Session 1 was conducted from April to May 2020 to collect participants' self-report PTSS and related questionnaires. In total, 126 COVID-19 survivors (discharged from hospitals between February and March 2020) and 126 non-COVID-19 controls completed Session 1. The self-report assessment set included the posttraumatic stress disorder (PTSD) checklist for DSM-5 (PCL-5), General Anxiety Disorder-7 (GAD-7), and Patient Health Questionnaire-9 (PHQ-9). Participants also reported their potential exposures during the pandemic (eTable 1 and eTable 2 in the Supplement).

Session 2 was conducted in August 2020 to collect brain imaging data, as well as the same set of self-report assessments administered in Session 1. In total, 50 COVID-19 survivors (discharged from hospitals between February and March 2020) and 43 non-COVID-19 controls completed Session 2. Data from three COVID-19 survivors were excluded from the analyses due to large artifacts of the MRI data. Of 47 COVID-19 survivors who were included in the analyses, 23 participated in both sessions. None of the controls in Session 2 participated in Session 1. The brain imaging data (i.e., structural and functional $\mathrm{MRl}$; $\mathrm{sMRI}$ and $\mathrm{fMRI}$ ) of all participants were collected at the radiology department of the Renmin Hospital of Wuhan University.

\section{Self-report assessments}

The PCL-5 was the primary self-report assessment in the present study. The PCL-5 consists of 20 items tapping into 20 symptoms of PTSD defined by DSM-5 [15], and all items are clustered into four subscales (i.e., intrusion, avoidance, cognition $/ \mathrm{mood}$, and arousal/reactivity). Each item is rated on a 5-point Likert scale (i.e., $0-4$ ). The total score of PCL-5 was the primary measure, and four subscale scores in PCL-5 were secondary measures. Participants also completed GAD-7 [16] and PHQ-9 [17] to measure their anxiety and depression levels, respectively. Based on the current participants, Cronbach's a for PCL-5 was 0.95 (for the patient group was 0.95 , and for the control group was 0.92), Cronbach's a for GAD-7 was 0.92 (for the patient group was 0.93 , and for the control group was 0.88), and Cronbach's a for PHQ-9 was 0.91 (for the patient group was 0.91, and for the control group was 0.87 ), which demonstrated the reliability of our selfreport assessments.

\section{MRI data acquisition}

MRI data were acquired using a GE 3.0 Tesla MR750 scanner with a standard 32-channel head coil. Subjects were asked to stay awake and to keep their heads still during the scan, with their eyes open and ears plugged. High resolution brain structural images were acquired with a T1weighted fast-spoiled gradient echo sequence (repetition time $=8.16 \mathrm{~ms}$, echo time $=3.18 \mathrm{~ms}$, flip angle $=12^{\circ}$, slice thickness $=1 \mathrm{~mm}$, interslice gap $=1 \mathrm{~mm}$, voxel size $=3 \mathrm{~mm} \times 3 \mathrm{~mm} \times 3 \mathrm{~mm}$, and $\mathrm{FOV}=256 \mathrm{~mm}$ ). Eight-minute resting-state brain functional images were acquired with a T2-weighted gradient echo planar imaging (repetition time $=2000 \mathrm{~ms}$, echo time $=30 \mathrm{~ms}$, flip angle $=90^{\circ}$, slice thickness $=3.5 \mathrm{~mm}$, interslice gap $=3.5 \mathrm{~mm}, \mathrm{FOV}=224 \mathrm{~mm}$, and 38 slices).

\section{MRI data analyses}

The main outcomes of interest in the MRI data analyses were (1) the brain structural volumes and (2) functional activity of key brain regions (i.e., bilateral hippocampus and amygdala).

Structural MRI images were analyzed using the Computational Anatomy Toolbox (CAT12; http://www.neuro.uni-jena.de/cat/) in SPM12. Data preprocessing included bias-field and noise removal, skull stripping, segmentation into gray and white matter, and normalization to Montreal Neurological Institute (MNI) space. The quality of images was assessed with the built-in image-quality rating and manually checked by the authors. Gray matter was spatially smoothed using the $8 \mathrm{~mm}$ full-width at halfmaximum (FWHM) Gaussian kernel. After preprocessing, we performed the voxel-based morphometry analysis [18] using the CAT12 toolbox and voxel-wise estimation of gray matter volume (GMV) for the whole brain of each participant.
Resting-state fMRI signals were preprocessed using the CONN toolbox version 19c (https://www.nitrc.org/projects/conn). The first ten scans were removed for signal equilibrium and participants' adaptation to the scanner's noise. Preprocessing steps included a standard pipeline (functional realignment and unwarp, functional slice-timing correction, structural unified segmentation and normalization, functional normalization, functional outlier detection, and functional smoothing with a $8 \mathrm{~mm}$ FWHM Gaussian kernel). The ART algorithm (http://www.nitrc.org/projects/ artifact_detect/) was applied to detect motion during the resting-state fMRI scan. Time points in subjects' images were marked as outliers if the global signal exceeded three standard deviations from the mean or if scanto-scan motion deviation exceeded $0.5 \mathrm{~mm}$. Those outliers, in addition to the linear and polynomial trends of six head motion parameters, were included as nuisance regressors during the denoising procedure in the CONN toolbox. Please note that we did not perform global signal regression in the analysis, since with and without global signal regression yielded similar statistical results in the present study. After preprocessing, we estimated voxel-wise amplitude of low-frequency fluctuation (ALFF) maps, which quantify the regional intensity of spontaneous fluctuations in fMRI blood oxygen level-dependent signals by calculating the square root of power spectrum within the low-frequency range $(0.01-0.08 \mathrm{~Hz})$ [19] using the CONN toolbox. Therefore, we used GMV and ALFF to quantify structures and functions of localized brain regions, respectively.

\section{Statistical analyses}

Statistical analyses for self-report data. In Session 1, self-report scores were compared using a two-way analysis of covariance (ANCOVA), with group (i.e., COVID-19 survivors and controls) and sex (i.e., males and females) as the fixed factors, and age as the covariate. When the group-sex interaction was significant, post-hoc independent-sample $t$ tests were performed, and $p$ values were Bonferroni corrected. ANCOVA was performed separated for different self-report assessments. The associations between the PCL-5 total score and the duration of hospitalization as well as the duration after discharge for COVID-19 survivors were assessed using the partial correlation analyses, with age and sex as covariates.

In Session 2, self-report scores were compared using a one-way ANCOVA, with group (i.e., COVID-19 survivors and controls) as the fixed factor, and age and sex as the covariates. Sex was omitted as a fixed factor in the analysis due to the small number of males for both groups $(N=14$ and $N=11$ for COVID-19 survivors and controls, respectively). For the 23 COVID-19 survivors who participated in both sessions, the changes of their PCL-5 total score were assessed using a paired-sample $t$ test, and the association between the changes of PCL-5 total score and the duration between two sessions was assessed using the partial correlation analysis, with age as the covariate.

Statistical analyses for MRI data. Mass univariate independent-sample $t$ tests were conducted to compare the group differences of voxel-wise GMV, adjusted by total intracranial volume (TIV), age, and sex, resulting in a map of $\mathrm{t}$ statistics and their associated $p$ values. The significance threshold was set as $p<0.005$ at the voxel level and $p_{\mathrm{FDR}}<0.05$ at the cluster level (false discovery rate correction was performed for multiple comparisons). Given the adjacency of hippocampus and amygdala, we applied the bilateral hippocampus and amygdala masks from the automated anatomical labeling atlas to isolate these two important structures in the cluster and report their respective peak coordinates/sizes.

After identifying brain regions that were significantly differed in the GMV between COVID-19 survivors and controls, ALFF values within each of these regions were extracted and compared between the two groups using the independent-sample $t$ test.

Finally, the associations between the MRI measures (i.e., GMV and ALFF) in the identified brain regions and the PCL-5 total score for COVID-19 survivors were assessed using the partial correlation analyses, with age and TIV as covariates.

\section{RESULTS}

\section{Self-report results in session 1}

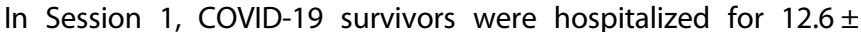
5.0 days and completed the self-report assessments $50.6 \pm$ 13.2 days after discharge (Table 1 ). The age $\left[t_{250}=0.24\right.$, $p=0.81]$ and the sex-ratio $\left(x^{2}=0, p=1.00\right)$ were not significantly different between COVID-19 survivors (mean \pm SD age, $52.4 \pm 13.5$ 
years; 40 males and 86 females) and controls (mean \pm SD age, 52.0 \pm 13.3 years; 40 males and 86 females).

ANCOVA results showed main effects of group $\left(F_{1,247}=23.5\right.$, $\left.p<0.001, \eta^{2}=0.09\right)$ and $\operatorname{sex}\left(F_{1}, 247=5.5, p=0.02, \eta^{2}=0.02\right)$, without a significant interaction effect $\left(F_{1,247}=0.56, p=0.46\right.$, $\eta^{2}=0.002$ ), on PCL-5 total score (Table 2; Fig. 1A). COVID-19 survivors had significantly higher PCL-5 total scores than controls (COVID-19 survivors: $11.8 \pm 11.9$; controls: $5.8 \pm 6.9$; difference, 6.3 [95\% Cl, 3.8-8.9]; $\left.t_{250}=4.9, p<0.001\right)$, and females had significantly higher PCL-5 total scores than males (females: $9.8 \pm 10.0$; males: $6.7 \pm 10.2$; difference, $3.1[95 \% \mathrm{Cl}, 0.5$ to 5.7$] ; t_{250}=2.4$, $p=0.02$ ). Moreover, the PCL-5 total score of COVID-19 survivors

Table 1. Demographics and clinical characteristics of COVID-19 survivors and controls in Session 1.

\begin{tabular}{|c|c|c|}
\hline \multirow[t]{2}{*}{ Characteristics } & \multicolumn{2}{|l|}{ Session 1} \\
\hline & $\begin{array}{l}\text { Covid-19 } \\
\text { survivors }\end{array}$ & Controls \\
\hline Age (years, SD) & $52.4 \pm 13.5$ & $52.0 \pm 13.3$ \\
\hline Sex (female/male) & $86 / 40$ & $86 / 40$ \\
\hline Clinical records ( $n, \%)$ & $126,100 \%$ & \\
\hline Fever $(n, \%)$ & $81,64 \%$ & \\
\hline Cough $(n, \%)$ & $69,55 \%$ & \\
\hline Hospitalization duration (days) & $12.6 \pm 5.0$ & \\
\hline \multicolumn{3}{|l|}{ Underlying diseases, (n \%) } \\
\hline Hypertension & $29,23 \%$ & \\
\hline Diabetes & $8,6 \%$ & \\
\hline \multicolumn{3}{|l|}{ Clinical type (n, \%) } \\
\hline Mild type & $117,93 \%$ & \\
\hline Severe type & $9,7 \%$ & \\
\hline Critical type & $0,0 \%$ & \\
\hline \multicolumn{3}{|l|}{ Treatment (n, \%) } \\
\hline Oxygen therapy & $26,21 \%$ & \\
\hline Anti-viral therapy & $81,64 \%$ & \\
\hline \multicolumn{3}{|l|}{ Potential exposure (n, \%) } \\
\hline Close people had Covid-19 & $74,59 \%$ & $15,12 \%$ \\
\hline Received intensive care & $12,10 \%$ & $0,0 \%$ \\
\hline Witnessed death & $33,26 \%$ & $2,2 \%$ \\
\hline $\begin{array}{l}\text { Witnessed others receive } \\
\text { intensive care }\end{array}$ & $32,25 \%$ & $2,2 \%$ \\
\hline Hopeless feeling & $92,73 \%$ & $37,29 \%$ \\
\hline
\end{tabular}

was significantly positively correlated with the duration from discharge to the date of participating in Session 1 (Fig. $1 \mathrm{~B} ; r=0.27$, $p=0.003)$, but not correlated with the duration of hospitalization $(r=-0.13, p=0.14)$.

For four subscales of PCL-5 and the total scores of GAD and $\mathrm{PHQ}$, significant group differences were identified (COVID19 survivors > controls) (Table 2). Moreover, females had significantly higher scores in the PCL-arousal, GAD-7, and PHQ-9 than males (Table 2).

\section{Self-report results in Session 2}

In Session 2, the age $\left(t_{250}=0.27, p=0.79\right)$ and sex-ratio $\left(X_{(1,90)}^{2}=0.20, p=0.66\right)$ were not significantly different between COVID-19 survivors (mean \pm SD age, $51.8 \pm 11.3$ years; 14 males and 33 females) and controls (mean \pm SD age, $52.5 \pm 11.0$ years; 11 males and 32 females). ANCOVA results revealed a significant main effect of group $\left(F_{1,86}=17.8, p<0.001, \eta^{2}=0.16\right)$ on PCL-5 total score (Fig. $\left.2 A\right)$. COVID-19 survivors had significantly higher PCL-5 total scores than controls (COVID-19 survivors: $15.0 \pm 12.0$; controls: $7.0 \pm 5.6$; difference, 8.2 [95\% Cl, 4.4 to 12.1$] ; t_{88}=4.2, p<0.001$ ).

For the 23 COVID-19 survivors who participated in both sessions, the duration between the two sessions was $97.4 \pm$ 11.8 days. The PCL-5 total score of these COVID-19 survivors was increased by $\sim 20 \%$, although not significant (from $10.8 \pm 8.1$ to $13.0 \pm 9.8$; difference, $2.1[95 \% \mathrm{Cl},-1.3$ to 5.6$] ; \mathrm{t}_{22}=1.29, p=0.21$ ) (Fig. 2B). Moreover, the changes of the PCL-5 total score were significantly positively correlated with the duration between the two sessions ( $r=0.46, p=0.03$; Fig. 2B).

\section{MRI results in Session 2}

COVID-19 survivors had significantly higher GMVs in the left amygdala (peak coordinate: $x=-23, y=0, z=-27$; peak $t$ value $=3.46$; cluster size $=66$ ), left hippocampus (peak coordinate: $x=-30, y=-8, z=$ -18 ; peak $t$ value $=3.39$; cluster size $=84$ ), right amygdala (peak coordinate: $x=27, y=0, z=-27$; peak $t$ value $=3.36$; cluster size $=$ 46), and right hippocampus (peak coordinate: $x=30, y=-14, z=$ -20 ; peak $t$ value $=3.41$; cluster size $=329$ ) (Fig. 2C). The volumes of the left amygdala and left hippocampus were significantly negatively correlated with the PCL-5 total score in COVID-19 survivors $(r=-0.37$, $p=0.01$ for left amygdala; $r=-0.31, p=0.04$ for left hippocampus; Fig. 2C) but not in controls ( $r=-0.08, p=0.62$ for left amygdala; $r=$ $-0.10, p=0.53$ for left hippocampus). The functional brain activities, represented as ALFF (Fig. 2D), were also significantly higher in COVID19 survivors as compared to controls in the left amygdala $\left(t_{88}=2.65\right.$, $p=0.01$, Cohen's $d=0.56$ ), left hippocampus ( $t_{88}=3.13, p=0.002$, Cohen's $d=0.67)$, right amygdala ( $t_{88}=2.92, p=0.005$, Cohen's $d=$ 0.62 ), and right hippocampus ( $t_{88}=2.90, p=0.005$, Cohen's $d=0.61$ ).

Table 2. Descriptive statistics and group-sex effect in self-report scores in Session 1.

\begin{tabular}{|c|c|c|c|c|c|c|c|c|c|c|c|c|c|c|}
\hline & \multirow{2}{*}{\multicolumn{2}{|c|}{$\begin{array}{l}\begin{array}{l}\text { Female } \\
\text { controls }\end{array} \\
(n=86)\end{array}$}} & \multirow{2}{*}{\multicolumn{2}{|c|}{$\begin{array}{l}\begin{array}{l}\text { Male } \\
\text { controls }\end{array} \\
(n=40)\end{array}$}} & \multirow{2}{*}{\multicolumn{2}{|c|}{$\begin{array}{l}\begin{array}{l}\text { Female } \\
\text { survivors }\end{array} \\
(n=86)\end{array}$}} & \multirow{2}{*}{\multicolumn{2}{|c|}{$\begin{array}{l}\begin{array}{l}\text { Male } \\
\text { survivors }\end{array} \\
(n=40)\end{array}$}} & \multicolumn{6}{|c|}{ ANCOVA } \\
\hline & & & & & & & & & \multicolumn{2}{|c|}{ Group } & \multicolumn{2}{|l|}{ Sex } & \multicolumn{2}{|c|}{ Interaction } \\
\hline PCL-total & 7.1 & 7.8 & 3.1 & 3.4 & 12.5 & 11.3 & 10.4 & 13.1 & 23.5 & $<0.001$ & 5.5 & 0.020 & 0.56 & 0.455 \\
\hline PCL-intrusion & 1.9 & 2.3 & 0.9 & 1.0 & 2.5 & 3.3 & 2.1 & 3.5 & 6.2 & 0.014 & 2.6 & 0.110 & 0.96 & 0.329 \\
\hline PCL-avoidance & 0.5 & 1.0 & 0.1 & 0.5 & 0.9 & 1.6 & 0.8 & 1.5 & 10.1 & 0.002 & 1.7 & 0.196 & 0.59 & 0.443 \\
\hline PCL-arousal/reactivity & 2.5 & 2.8 & 1.2 & 1.5 & 4.6 & 3.7 & 3.6 & 4.3 & 25.8 & $<0.001$ & 7.6 & 0.006 & 0.07 & 0.799 \\
\hline GAD & 3.4 & 3.1 & 1.6 & 2.3 & 5.8 & 4.6 & 4.0 & 4.5 & 22.5 & $<0.001$ & 13.5 & $<0.001$ & 0.01 & 0.913 \\
\hline PHQ & 3.7 & 3.6 & 1.9 & 2.6 & 6.5 & 5.1 & 4.9 & 5.1 & 25.4 & $<0.001$ & 11.0 & 0.001 & 0.01 & 0.909 \\
\hline
\end{tabular}

$\mathrm{PCL}=$ Posttraumatic-Stress Disorder (PTSD) Checklist for DSM-5; GAD = total score for the items of Generalized Anxiety Disorder Screener; PHQ = total score for the items of Patient Health Questionnaire-Depression Module (PHQ). 

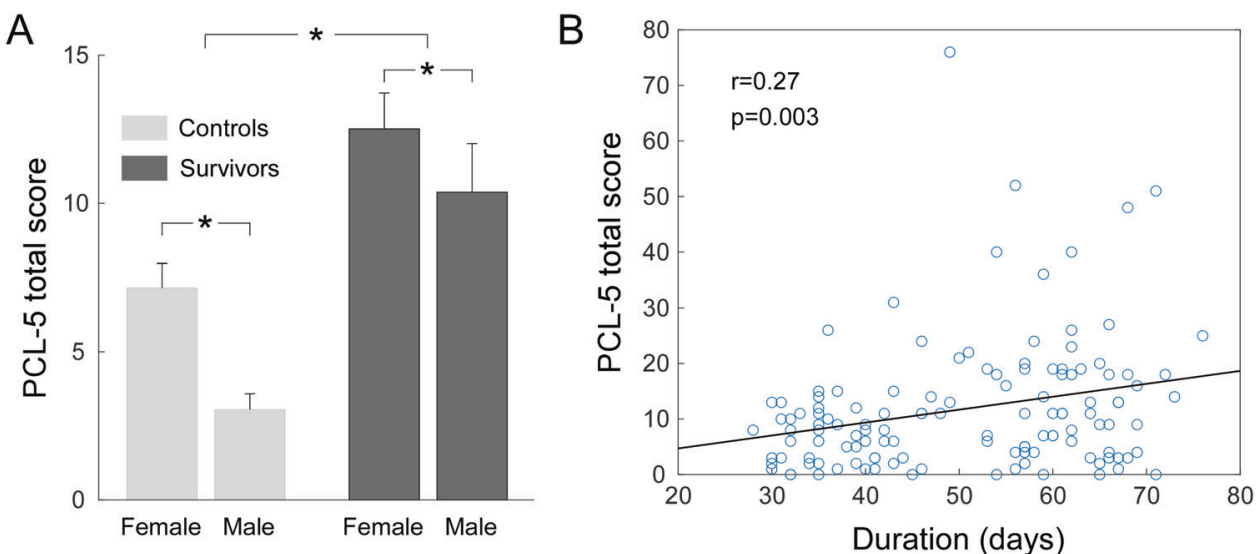

Fig. 1 PCL-5 total score of COVID-19 survivors and controls in Session 1. A. COVID-19 survivors had significantly higher scores than controls, and females had significantly higher scores than males. B. The score of COVID-19 survivors was significantly positively correlated with the duration from discharge to the date of participating in Session 1. Error bars represent the standard error of the mean. Asterisks represent $p<0.05$.

\section{DISCUSSION}

In this two-session follow-up study, we assessed PTSS of COVID-19 survivors in Wuhan after they were discharged from hospitals for about 3-6 months. The current results suggested that (1) compared to non-COVID-19 controls, COVID-19 survivors self-reported more PTSS at both sessions; (2) the severity of PTSS in COVID-19 survivors was correlated with the duration after they were discharged (i.e., $r=$ $0.27, p=0.003$, in Session 1), and increased along with the days between two sessions (i.e., the increase rate was around 20\% from Session 1 to Session 2); and (3) females self-reported more PTSS than males. Brain imaging results suggested that COVID-19 survivors had larger GMVs and higher functional activities in bilateral hippocampus and amygdala, as compared to controls. However, the GMVs of the left hippocampus and amygdala were negatively correlated with the severity of PTSS for COVID-19 survivors.

A recent study demonstrated that severe PTSS could be a disabling short-term consequence (i.e., within one month) for COVID-19 survivors after they were discharged from hospitals [9]. Beyond this finding, we showed that COVID-19 survivors had significantly higher PCL-5 scores than non-COVID-19 community dwellers for a longer period (about 3-6 months) after discharge. Crucially, the PCL-5 total scores of COVID-19 survivors were significantly positively correlated with the duration from discharge to the date of assessment in Session 1. Moreover, as per COVID-19 survivors who participated in both sessions, their total scores of PCL-5 increased by $\sim 20 \%$, and the increases were positively correlated with the duration between two sessions. Our results highlight that COVID-19 survivors may develop more severe PTSS without early intervention. These results contrasted the previous findings based on the general public; as per the general populations, results showed a statistically significant longitudinal reduction of PTSS after COVID-19 outbreak [20]. As note, COVID-19 survivors might experience social isolation, discrimination, unemployment, and economic losses in a long time after they were discharged from hospitals. Moreover, since COVID-19 is still an ongoing pandemic, they might still in an acute stress state from a long-term perspective. Therefore, their mental health problems may show a different trajectory as the general public.

Importantly, we also observed that females (regardless if they were COVID-19 survivors or controls) suffered more PTSS than males. This finding is in line with previous epidemiological studies of mental health on healthcare workers and the general populations during the COVID-19 pandemic [21, 22]. Essentially, regardless of being infected by the virus or not, females had higher rates of affective symptoms compared to males and may develop more mental health problems during the pandemic.
Governments and relevant social authorities need to consider providing additional care for females during the pandemic.

The amygdala and hippocampus are the two most well-studied brain regions that may be involved in the pathophysiology of PTSS [11]. The amygdala is critical in the process of fear response, conditioning, and generalization [23], and facilitates responses to stressful experience [24]. Hippocampus interacts with the amygdala during the encoding of emotional memories, which is highly relevant to trauma and PTSS [25]. Previous neuroimaging studies frequently reported that the volumes of the amygdala and hippocampus were smaller in patients suffering from PTSS (e.g., combat veterans or individuals with childhood trauma) $[12,13,26,27]$. Nevertheless, the current results suggested that both the GMVs and functional brain activities in bilateral amygdala and hippocampus were significantly larger in COVID-19 survivors. In contrast to literatures studying past trauma, COVID-19 pandemic should be considered as an ongoing traumatic event as the elevated anxiety and stress levels have been noted during both the severe and remission stages [28]. In other words, COVID19 survivors might still experience negative feelings (e.g., fear and anger). Hence, the amygdala and hippocampus could be still hyper-activated among COVID-19 survivors, and the enlarged structural volumes of these brain regions could be a functional compensation to cope with the acute stress and the ongoing COVID-19 related trauma [29]. By looking into the relationship of GMV and severity of PTSS, we found negative correlations for the left amygdala and hippocampus in COVID-19 survivors but not in controls. This result was consistent with previous observations of the neurological impairments in the amygdala and hippocampus in patients suffering from PTSS [13, 30, 31].

This study has several limitations. First, we only followed up a short to medium period. The PTSS course of COVID-19 survivors in long terms needs further investigation. Second, we only recruited participants (i.e., COVID-19 survivors in both sessions) from Wuhan (i.e., the center of outbreak at the beginning of 2020). Cohort studies from other cities and countries are necessary for validation. Third, only 23 COVID-19 survivors participated in both sessions. Therefore, the trajectory of PTSS in COVID-19 survivors requires further confirmation by studies with a larger sample size. Fourth, we did not consider gender effect in the MRI analysis due to the small number of males for both groups. Last, we could not completely rule out the possible effects of COVID-19 infection and medications on abnormal brain structures.

In conclusion, our results suggest that COVID-19 survivors have been coping with PTSS, and the severity of PTSS may be aggravated after discharge. The present study highlights the importance of 
A

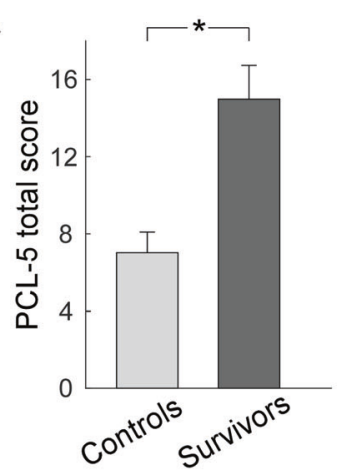

B

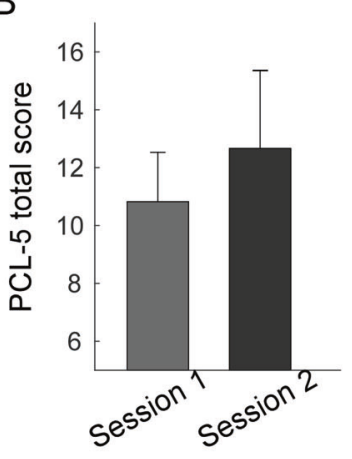

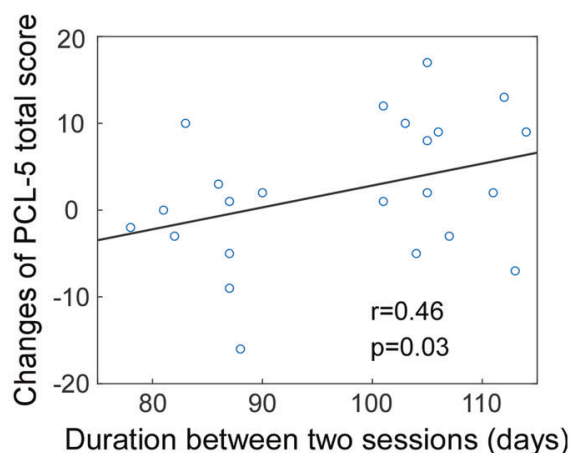

C
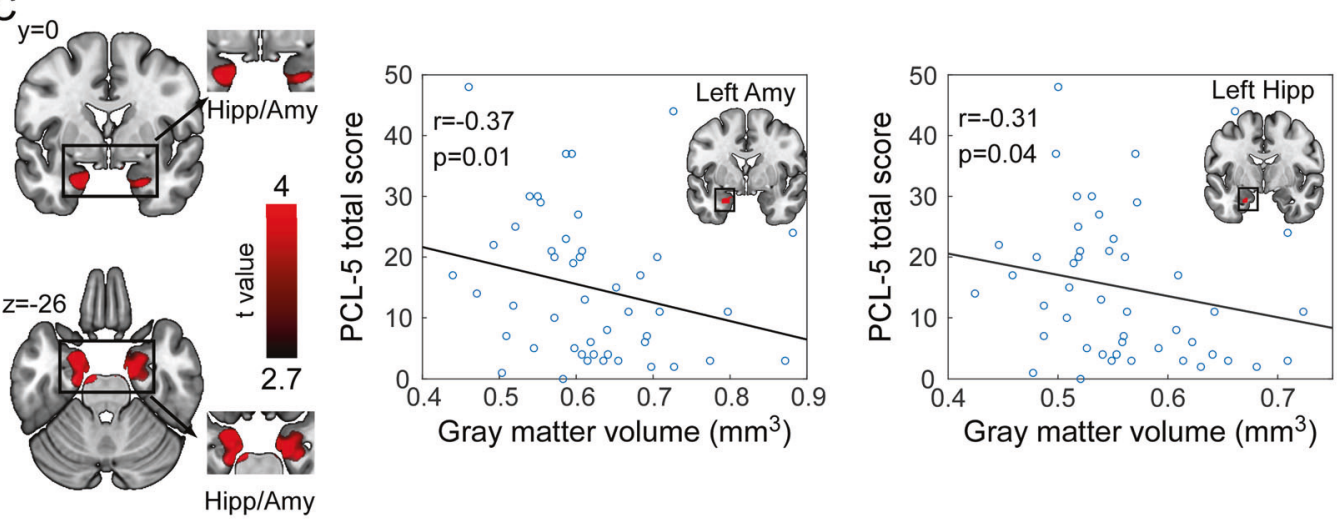

D

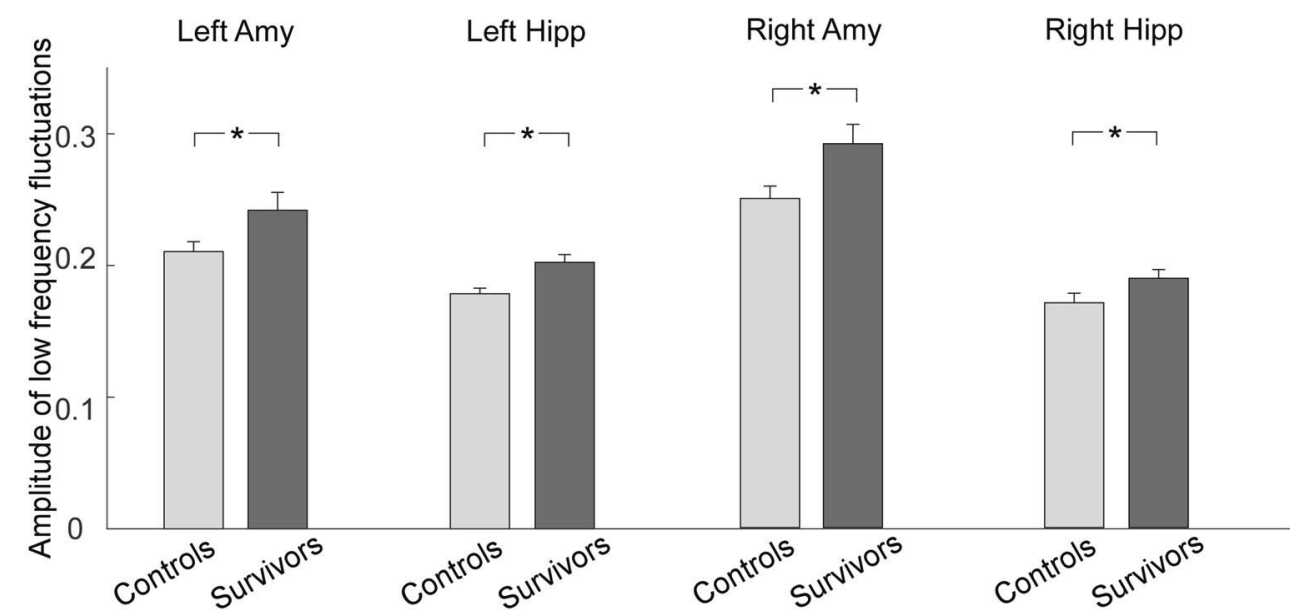

Fig. 2 Self-report and MRI results in Session 2. A. COVID-19 survivors had significantly higher PCL-5 total scores than controls. B. For the 23 COVID-19 survivors completed both sessions, the PCL-5 total score was increased by approximately $20 \%$ from Session 1 to Session 2 . The changes of the score were significantly positively correlated with the duration between the two sessions. C. COVID-19 survivors had significantly higher gray matter volumes in bilateral hippocampus and amygdala than controls. The volumes of the left amygdala and left hippocampus were significantly negatively correlated with the PCL-5 total scores. D. ALFF values in bilateral hippocampus and amygdala were significantly higher in COVID-19 survivors than controls. Hipp: hippocampus, Amy: amygdala. Error bars represent the standard error of the mean. Asterisks represent $p<0.05$.

monitoring the mental wellness of COVID-19 survivors and calls for mental supports and early intervention.

\section{CODE AVAILABILITY}

The codes for analyzing MRI data can be found in the CAT12 toolbox (http://www. neuro.uni-jena.de/cat/) and CONN toolbox (version 17f, https://www.nitrc.org/ projects/conn). No customized codes were used in this study.

\section{DATA AVAILABILITY}

Data in the present study can be request from the corresponding author with clear research aims.

\section{REFERENCES}

1. Zhu N, Zhang D, Wang W, Li X, Yang B, Song J, et al. A novel coronavirus from patients with pneumonia in China, 2019. N Engl J Med. 2020;382:727-733.

2. Paterson RW, Brown RL, Benjamin L, Nortley R, Wiethoff S, Bharucha T, et al. The emerging spectrum of COVID-19 neurology: Clinical, radiological and laboratory findings. Brain. 2020;143:3104-3120.

3. Pezzini A, Padovani A. Lifting the mask on neurological manifestations of COVID19. Nat Rev Neurol. 2020;16:636-644.

4. Kandemirli SG, Dogan L, Sarikaya ZT, Kara S, Akinci C, Kaya D, et al. Brain MRI findings in patients in the intensive care unit with COVID-19 infection. Radiology. 2020;297:E232-E235. 
5. Egbert AR, Cankurtaran S, Karpiak S. Brain abnormalities in COVID-19 acute/ subacute phase: a rapid systematic review. Brain Behav Immun. 2020;89:543-554.

6. Duanan K, Premi E, Pilotto A, Cristillo V, Benussi A, Libri I, et al. Alterations of frontal-temporal gray matter volume associate with clinical measures of older adults with COVID-19. Neurobiol Stress. 2021;14:100326.

7. Huang C, Huang L, Wang Y, Li X, Ren L, Gu X, et al. 6-month consequences of COVID-19 in patients discharged from hospital: a cohort study. Lancet. 2021;397:220-232.

8. Zhou Y, Sun Z, Wang Y, Xing C, Sun L, Shang Z, et al. The prevalence of PTSS under the influence of public health emergencies in last two decades: a systematic review and meta-analysis. Clin Psychol Rev. 2021;83:101938.

9. Mazza MG, De Lorenzo R, Conte C, Poletti S, Vai B, Bollettini I, et al. Anxiety and depression in COVID-19 survivors: role of inflammatory and clinical predictors. Brain Behav Immun. 2020;89:594-600.

10. Pfefferbaum B, North CS. Mental health and the Covid-19 pandemic. N Engl J Med. 2020;383:510-512.

11. Shin LM, Rauch SL, Pitman RK. Amygdala, medial prefrontal cortex, and hippocampal function in PTSD. Ann NY Acad Sci. vol. 1071, Blackwell Publishing Inc.; 2006. p. 67-79.

12. Wang Z, Neylan TC, Mueller SG, Lenoci M, Truran D, Marmar CR, et al. Magnetic resonance imaging of hippocampal subfields in posttraumatic stress disorder. Arch Gen Psychiatry. 2010;67:296-303.

13. Morey RA, Gold AL, LaBar KS, Beall SK, Brown VM, Haswell CC, et al. Amygdala volume changes in posttraumatic stress disorder in a large case-controlled veterans group. Arch Gen Psychiatry. 2012;69:1169-1178.

14. Clinical management of COVID-19. https://www.who.int/publications/i/item/ clinical-management-of-covid-19 Accessed 1 Jan 2021.

15. Blevins CA, Weathers FW, Davis MT, Witte TK, Domino JL. The Posttraumatic Stress Disorder Checklist for DSM-5 (PCL-5): development and initial psychometric evaluation. J Trauma Stress. 2015;28:489-498.

16. Spitzer RL, Kroenke K, Williams JBW, Löwe B. A brief measure for assessing generalized anxiety disorder: The GAD-7. Arch Intern Med. 2006;166:1092-1097.

17. Kroenke K, Spitzer RL, Williams JBW. The PHQ-9: validity of a brief depression severity measure. J Gen Intern Med. 2001;16:606-613.

18. Ashburner J, Friston K. Unified segmentation. Neuroimage 2005;26:839-851.

19. Zang YF, He Y, Zhu CZ, Cao QJ, Sui MQ, Liang M, et al. Altered baseline brain activity in children with ADHD revealed by resting-state functional MRI. Brain Dev. 2007;29:83-91.

20. Wang C, Pan R, Wan X, Tan Y, Xu L, Mclntyre RS, et al. A longitudinal study on the mental health of general population during the COVID-19 epidemic in China. Brain Behav Immun. 2020;87:40-48.

21. Vindegaard N, Benros ME. COVID-19 pandemic and mental health consequences: systematic review of the current evidence. Brain Behav Immun. 2020;89:531-542.

22. Pappa S, Ntella V, Giannakas T, Giannakoulis VG, Papoutsi E, Katsaounou P. Prevalence of depression, anxiety, and insomnia among healthcare workers during the COVID-19 pandemic: a systematic review and meta-analysis. Brain Behav Immun. 2020;88:901-907.

23. Dunsmoor JE, Prince SE, Murty VP, Kragel PA, LaBar KS. Neurobehavioral mechanisms of human fear generalization. Neuroimage. 2011;55:1878-1888.

24. McEwen BS. Physiology and neurobiology of stress and adaptation: central role of the brain. Physiol Rev. 2007;87:873-904.
25. McGaugh JL. The amygdala modulates the consolidation of memories of emotionally arousing experiences. Annu Rev Neurosci. 2004;27:1-28.

26. Karl A, Schaefer M, Malta LS, Dörfel D, Rohleder N, Werner A. A meta-analysis of structural brain abnormalities in PTSD. Neurosci Biobehav Rev. 2006;30: 1004-1031.

27. Vythilingam $M$, Heim $C$, Newport J, Miller AH, Anderson E, Bronen R, et al. Childhood trauma associated with smaller hippocampal volume in women with major depression. Am J Psychiatry. 2002;159:2072-2080.

28. He L, Wei D, Yang F, Zhang J, Cheng W, Feng J, et al. Functional connectome prediction of anxiety related to the COVID-19 pandemic. Am J Psychiatry. 2021;00:appi.ajp.2020.2.

29. Lu Y, Li X, Geng D, Mei N, Wu PY, Huang CC, et al. Cerebral micro-structural changes in COVID-19 patients-an MRI-based 3-month follow-up study: a brief title: cerebral changes in COVID-19. eClin. Med. 2020;25:100484.

30. Ousdal OT, Milde AM, Hafstad GS, Hodneland E, Dyb G, Craven AR, et al. The association of PTSD symptom severity with amygdala nuclei volumes in traumatized youths. Transl Psychiatry. 2020;10:1-10.

31. Logue MW, van Rooij SJH, Dennis EL, Davis SL, Hayes JP, Stevens JS, et al. Smaller hippocampal volume in posttraumatic stress disorder: a multisite ENIGMA-PGC study: subcortical volumetry results from Posttraumatic Stress Disorder Consortia. Biol Psychiatry. 2018;83:244-253.

\section{ACKNOWLEDGEMENTS}

This work was supported by the National Natural Science Foundation of China (31822025 and 32071061 to LH) and the Scientific Foundation of Institute of Psychology, Chinese Academy of Sciences (EOCX521003 to YT). We also thank the patients and families who contributed to these studies.

\section{COMPETING INTERESTS}

The authors declare no competing interests.

\section{ADDITIONAL INFORMATION}

Supplementary information The online version contains supplementary material available at https://doi.org/10.1038/s41380-021-01223-w.

Correspondence and requests for materials should be addressed to Z.L. or L.H.

Reprints and permission information is available at http://www.nature.com/ reprints

Publisher's note Springer Nature remains neutral with regard to jurisdictional claims in published maps and institutional affiliations. 